\title{
Residual tumor volume versus extent of resection: predictors of survival after surgery for glioblastoma
}

\author{
Clinical article
}

\author{
Matthew M. Grabowski, B.S., ${ }^{1}$ Pablo F. Recinos, M.D., ${ }^{1}$ Amy S. Nowacki, Ph.D., ${ }^{2}$ \\ Jason L. Schroeder, M.D., ${ }^{1}$ Lilyana Angelov, M.D., ${ }^{1}$ Gene H. Barnett, M.D., M.B.A., ${ }^{1}$ \\ and Michael A. Vogelbaum, M.D., Ph.D. ${ }^{1}$
}

${ }^{1}$ Brain Tumor and Neuro-Oncology Center and Department of Neurosurgery, and ${ }^{2}$ Department of Quantitative Health Sciences, Cleveland Clinic, Cleveland, Ohio

\begin{abstract}
Object. The impact of extent of resection (EOR) on survival for patients with glioblastoma (GBM) continues to be a point of debate despite multiple studies demonstrating that increasing EOR likely extends survival for these patients. In addition, contrast-enhancing residual tumor volume (CE-RTV) alone has rarely been analyzed quantitatively to determine if it is a predictor of outcome. The purpose of this study was to evaluate the effect of CE-RTV and T2/FLAIR residual volume (T2/F-RV) on overall survival.

Methods. A retrospective review of 128 patients who underwent primary resection of supratentorial GBM followed by standard radiation/chemotherapy was undertaken utilizing quantitative, volumetric analysis of pre- and postoperative MR images. The results were compared with clinical data obtained from the patients' medical records.

Results. At analysis, $8 \%$ of patients were alive, and no patients were lost to follow-up. The overall median survival was 13.8 months, with a median Karnofsky Performance Scale (KPS) score of 90 at presentation. The median contrast-enhancing preoperative tumor volume (CE-PTV) was $29.0 \mathrm{~cm}^{3}$, and CE-RTV was $1.2 \mathrm{~cm}^{3}$, equating to a 95.8\% median EOR. The median T2/F-RV was $36.8 \mathrm{~cm}^{3}$. CE-PTV, CE-RTV, T2/F-RV, and EOR were all statistically significant predictors of survival when controlling for age and KPS score. A statistically significant benefit in survival was seen with a CE-RTV less than $2 \mathrm{~cm}^{3}$ or an EOR greater than $98 \%$. Evaluation of the volumetric analysis methodology was performed by observers of varying degrees of experience - an attending neurosurgeon, a fellow, and a medical student. Both the medical student and fellow recorded correlation coefficients of 0.98 when compared with the attending surgeon's measured volumes of CE-PTV, while for CE-RTV, correlation coefficients of 0.67 and 0.71 (medical student and fellow, respectively) were obtained.

Conclusions. CE-RTV and EOR were found to be significant predictors of survival after GBM resection. CERTV was the more significant predictor of survival compared with EOR, suggesting that the volume of residual contrast-enhancing tumor may be a more accurate and meaningful reflection of the pathobiology of GBM.

(http://thejns.org/doi/abs/10.3171/2014.7.JNS132449)
\end{abstract}

$\begin{array}{llllll}\text { KEY WORDS } & \bullet & \text { glioblastoma } \\ \text { volumetrics } & \bullet & \text { oncology }\end{array}$

$\mathrm{G}$ LIOBLASTOMA (GBM) is the highest-grade, primary central nervous system cancer and has a 5-year survival rate of less than $5 \% .{ }^{4}$ Current guidelines for the treatment of GBM call for a combination of resection, radiation therapy, and chemotherapy to improve

\footnotetext{
Abbreviations used in this paper: $\mathrm{AIC}=$ Akaike information criterion; $\mathrm{AN}=$ attending neurosurgeon; $\mathrm{CE}-\mathrm{PTV}=$ contrast-enhancing preoperative tumor volume; CE-RTV = contrast-enhancing residual tumor volume; EOR = extent of resection; FEL = fellow; FLAIR = fluid-attenuated inversion recovery; GBM = glioblastoma; KPS = Karnofsky Performance Scale; MPRAGE = magnetization-prepared rapid acquisition gradient echo; $\mathrm{MS}=$ medical student; $\mathrm{PTV}=$ preoperative tumor volume; SSDI = Social Security Death Index; $\mathrm{T} 2 / \mathrm{F}-\mathrm{RV}=\mathrm{T} 2 / \mathrm{FLAIR}$ residual volume.
}

symptoms and prolong lifespan. ${ }^{19,22}$ Several prognostic factors have been identified and prospectively validated as indicators of shorter survival in patients with highgrade gliomas, including advanced age, aggressive histological features, and low Karnofsky Performance Scale (KPS) scores. ${ }^{3,5,22}$ While most within the field of neurooncology agree that maximal resection of the tumor offers the best chance for prolonged survival, the impact of extent of resection (EOR) on survival continues to be a point of discussion..$^{15}$

Previous studies have used both CT and MRI to try

This article contains some figures that are displayed in color online but in black-and-white in the print edition. 
to determine EOR and its implications for survival in GBM patients. Although there are no definitive prospective studies that have shown that EOR alone alters survival, there is a substantial volume of retrospective literature that has shown that maximizing EOR likely extends time to progression and increases survival. 2,7,12-14,16 However, there are inconsistencies in the way that we evaluate EOR in gliomas. In the case of low-grade gliomas, the volume of T2 or FLAIR abnormality is used to delineate tumor burden and completeness of resection, whereas for highgrade gliomas, only contrast-enhancing volume is typically used and the T2/FLAIR components of the tumor are typically ignored. ${ }^{21,23}$ Given that brain infiltrated with tumor does not always enhance, but still may appear abnormal on other MRI sequences, the contrast-enhancing tumor volume may underestimate the full extent of tumor burden..$^{10,23}$

Extent of resection is not an ideal measure of the success of surgery, as it does not convey a direct indication of the residual disease burden that must be addressed by medical therapies (i.e., radiation therapy and chemotherapy). The biological impact of EOR is likely to be strongly dependent upon the preoperative tumor volume (PTV). That is, a near-total resection of a very large tumor may leave behind more tumor mass than a subtotal resection of a small tumor, and yet the proposed relationship between EOR and outcome would suggest that it is the more complete resection that conveys a better prognosis, regardless of residual tumor burden. Furthermore, past studies have neglected to evaluate the relationship between postsurgical T2/FLAIR residual volume (T2/F-RV), which is known to include infiltrating glioma cells, and clinical outcome. These inconsistencies, along with the methodological shortcomings of previous studies, raise questions about the biological relevance of past analyses. We hypothesized that the pathobiology of GBM is primarily dependent on residual tumor volume (RTV) rather than EOR and sought to evaluate the effect of both contrast-enhancing RTV (CE-RTV) and T2/F-RV on overall survival with use of volumetric analysis.

\section{Methods}

This study was approved by the institutional review board of the Cleveland Clinic.

\section{Patient Selection}

A retrospective study was performed of all cases of supratentorial GBM in adult patients who were treated at the Cleveland Clinic with surgery followed by standard radiation and chemotherapy ${ }^{19}$ between the years 2005 and 2010. Patients were included if they had a newly discovered, histologically confirmed GBM, and underwent resection, a preoperative MRI study, and a postoperative MRI study acquired no later than 48 hours after the time of surgery. Patients were excluded if they underwent a needle biopsy only or if they had previously been treated with chemotherapy and/or radiation prior to resection of the GBM.

All surgeries were performed with the use of image-guided navigation. A contrast-enhanced MPRAGE (magnetization-prepared rapid acquisition gradient echo) sequence, usually obtained the day before surgery, was used as the reference study for intraoperative navigation. No patient included in this analysis underwent surgery with the use of intraoperative MRI. Forty-three patients underwent intraoperative electrocorticography and/or awake monitoring.

An institutional review board-approved database was populated using demographic data, clinical outcomes, and treatment-related variables. Variables collected that were selected for analysis included age at diagnosis, KPS score, any adjuvant therapy (chemotherapy/radiation), and tumor location (lobe). Data were obtained from electronic medical records and mortality was cross-referenced using the Social Security Death Index (SSDI).

\section{Eloquence Definition}

Each tumor was defined as being in a noneloquent, near-eloquent, or eloquent region based on preoperative MRI scans and as described by Sawaya et al. ${ }^{17}$ and Lacroix et al. ${ }^{12}$ Lesions in noneloquent regions included frontal and temporal polar lesions, a right-sided parietooccipital lesion, or a lesion located in the cerebellar hemisphere. Eloquent regions included the motor/sensory cortex, visual or speech center, basal ganglia, hypothalamus, brainstem, and corpus callosum. Near-eloquent regions were defined as immediately adjacent to eloquent structures. Importance of nearby white matter tracts was not assessed.

\section{Volumetric Analysis}

The authors used a quantitative, semiautomated volumetric analysis tool (BrainLab prototype iPlan software) to measure PTV and RTV. The analysis was performed independently by 3 individuals who were blinded to each others' results (M.M.G., P.F.R., M.A.V.). The assessors included a medical student, a fellow, and a senior attending neurosurgeon with over 15 years of a dedicated neurosurgical oncology practice (M.A.V.), thus representing a wide range of experience in the field. Determination of volumes was made without consideration of clinical outcome. The volumes taken by the attending neurosurgeon were considered the standard measurements that were used for all statistical calculations aside from those related to interobserver variability and correlations. T2/F-RV was assessed by the attending neurosurgeon only.

All volumes were defined in three dimensions using preoperative and postoperative MR images obtained on 1.5-T scanners. Postoperative images were obtained within 48 hours from the time of surgery. Imaging sequences that were evaluated included pre- and postcontrast 3D MPRAGE and 2D precontrast T2/FLAIR sequences. Contrast-enhancing preoperative tumor volume (CEPTV) was defined as the volume of contrast-enhancing brain tissue for MPRAGE sequences. It is recognized that postoperative blood products, which are hyperintense in the acute phase on T1-weighted or MPRAGE sequences, and when localized within the area of enhancement of the contrast images, can inflate the apparent volume of the postoperative "enhancement." To avoid inclusion of hy- 
perintense blood or blood products from the RTV calculations, we evaluated the volume of hyperintense tissue on the postoperative, precontrast MPRAGE images and subtracted this overlapping volume from the subsequently determined volume for the postoperative, postcontrast MPRAGE images. Postoperative T2/F-RV was calculated as the volume of T2/FLAIR abnormality, with the resection cavity excluded. For this analysis, EOR was calculated using the following formula: [(PTV - RTV) / PTV] $\times 100 \%$.

\section{Survival Analysis}

The primary end point used in this analysis was overall survival, as calculated by the time from initial surgery to the date of death in months. The date of death was confirmed using the SSDI, while patients who had no entry in the SSDI and had recent clinical contact were considered alive and censored at the date of cross-reference with the SSDI in the analysis. Age at diagnosis (in years), KPS score, EOR (as a fraction), PTV and RTV (both in cubic centimeters) were analyzed as continuous variables.

\section{Statistical Analysis: Interobserver Variability and} Correlations

To assess interobserver variability between the medical student, fellow, and attending neurosurgeon, Pearson correlation coefficients and $\mathrm{p}$ values were reported for each variable and between each volume measurement taken by the attending neurosurgeon.

The following statistical tests used only the measurements taken by the attending neurosurgeon. Cox proportional hazards regression was used to investigate associations of clinical variables and volumetric measurements with survival. The candidate predictors of survival were identified based on the literature along with each of the volume measurements. The Akaike information criterion (AIC) was used to determine the best volumetric predictor of survival. The AIC is a measure of the relative quality of a statistical model for a given set of data. As such, it provides a means for model selection, dealing with the tradeoff between complexity of the model and goodness of fit of the model. Thus, AIC not only rewards goodness of fit, but also includes a penalty that is an increasing function of the number of estimated parameters (to avoid overfitting the model). Given a set of candidate models for the data, the preferred model is the one with the minimum AIC value (the absolute value of the AIC is of no consequence by itself).

\section{Results}

\section{Patient Characteristics}

A total of 128 patients met the inclusion criteria for this study, with an age range of 23-86 years (median 60 years). Their initial median KPS score was 90 (range 30-100). The median duration of survival (overall survival) was 13.8 months (range 1-84 months). At the time of analysis, $8 \%$ of patients were alive, and no patients had been lost to follow-up. Thirty-one patients had tumors located in eloquent areas of the brain, 48 in near- eloquent regions, and 49 in noneloquent regions. All patients received conventional chemoradiation followed by adjuvant chemotherapy, and most patients received some combination of adjuvant chemotherapies, investigational agent(s), and/or underwent subsequent resections at the time of recurrence. The immediate preoperative clinical parameters of the patients enrolled in this study are summarized in Table 1.

\section{Interobserver Correlations}

The Pearson correlation coefficients for the 3 observers' contrast-enhancing volume measurements are reported in Table 2. In defining CE-PTV, both the medical student and the fellow recorded correlation coefficients of 0.98 when compared with the standard measurements (attending surgeon's measured volumes). For CE-RTV, the fellow recorded a correlation coefficient of 0.71 when compared with the standard measurement, while the medical student recorded a 0.67 when similarly compared. Following this trend, correlation coefficients for

\section{TABLE 1: Patient characteristics*}

\begin{tabular}{|c|c|}
\hline Variable & Value \\
\hline no. of patients & 128 \\
\hline \multicolumn{2}{|l|}{ age } \\
\hline median & $60 \mathrm{yrs}$ \\
\hline range & $23-86$ yrs \\
\hline \multicolumn{2}{|l|}{ KPS } \\
\hline median & 90 \\
\hline range & $30-100$ \\
\hline \multicolumn{2}{|l|}{ overall survival } \\
\hline median & $13.8 \mathrm{mos}$ \\
\hline range & $1-84$ mos \\
\hline \multicolumn{2}{|c|}{ eloquence of tumor location } \\
\hline noneloquent & 49 \\
\hline eloquent & 31 \\
\hline near-eloquent & 48 \\
\hline \multicolumn{2}{|l|}{ CE-PTV $†$} \\
\hline median & $29.0 \mathrm{~cm}^{3}$ \\
\hline range & $0.3-121.6 \mathrm{~cm}^{3}$ \\
\hline \multicolumn{2}{|l|}{ CE-RTV† } \\
\hline median & $1.2 \mathrm{~cm}^{3}$ \\
\hline range & $0-30.5 \mathrm{~cm}^{3}$ \\
\hline \multicolumn{2}{|l|}{ EOR† } \\
\hline median & $95.8 \%$ \\
\hline range & $56.9-100 \%$ \\
\hline \multicolumn{2}{|l|}{$\mathrm{T} 2 / \mathrm{F}-\mathrm{RV} \dagger$} \\
\hline median & $36.75 \mathrm{~cm}^{3}$ \\
\hline range & $0.59-169.71 \mathrm{~cm}^{3}$ \\
\hline
\end{tabular}

* CE-PTV = contrast-enhancing preoperative tumor volume; CE-RTV = contrast-enhancing residual tumor volume; $E O R=$ extent of resection; T2/F-RV = T2/FLAIR residual volume.

$\dagger$ All volumes defined from the attending neurosurgeon's measurements. 
M. M. Grabowski et al.

TABLE 2: Inter-reviewer correlations *

\begin{tabular}{lcc}
\hline Variable-Variable & r Value & p Value \\
\hline CE-PTV: MS-AN & 0.98 & $<0.0001$ \\
CE-PTV: FEL-AN & 0.98 & $<0.0001$ \\
CE-RTV: MS-AN & 0.67 & $<0.0001$ \\
CE-RTV: FEL-AN & 0.71 & $<0.0001$ \\
EOR: MS-AN & 0.70 & $<0.0001$ \\
EOR: FEL-AN & 0.71 & $<0.0001$ \\
\hline
\end{tabular}

* The attending neurosurgeon's measurements were the standard for this study. Boldface type indicates statistical significance. AN = attending neurosurgeon; $E O R$ = extent of resection; $F E L=$ fellow; $M S$ = medical student; PTV = preoperative tumor volume; RTV = residual tumor volume.

EOR measurements were 0.71 and 0.70 for the fellow and medical student, respectively, when compared with the standard measurement.

\section{Volume Measurement Correlations}

Some of the variables assessed are likely to be interdependent. For example, one could assume that a greater EOR should be associated with a smaller RTV. However, the relationship between EOR and RTV also depends upon the PTV. For any given percent resection, the RTV will increase with PTV. Hence, we decided to further evaluate the degree to which these variables are correlated. Correlations between the standard measurements of tumor volume are reported in Table 3. Variables with highly statistically significant correlations $(\mathrm{p}<0.0001)$ include the following: EOR and CE-RTV $(r=-0.71)$; CEPTV and CE-RTV $(r=0.41)$; CE-PTV and T2/F-RV $(r=$ $0.38)$. CE-RTV and T2/F-RV were weakly correlated ( $\mathrm{r}=$ $0.22, \mathrm{p}=0.01$ ), while EOR was not correlated with CEPTV or T2/F-RV ( $r=0.04$ and -0.07 , respectively).

\section{Predictors of Survival-T1-Weighted and FLAIR Images}

Median values for tumor volume measurements are reported in Table 1 . The median CE-PTV was $29.0 \mathrm{~cm}^{3}$ (range $0.3-121.6 \mathrm{~cm}^{3}$ ) and the median CE-RTV was 1.2 $\mathrm{cm}^{3}$ (range $0-30.5 \mathrm{~cm}^{3}$ ), equating to a $95.8 \%$ median EOR (range $56.9 \%-100 \%$ ). Median T2/F-RV was 36.8 $\mathrm{cm}^{3}$ (range $0.6-169.7 \mathrm{~cm}^{3}$ ).

TABLE 3: Volume measurement correlations*

\begin{tabular}{lcc}
\hline Variable-Variable & r Value & p Value \\
\hline CE-PTV-CE-RTV & 0.41 & $<0.0001$ \\
CE-PTV-T2/F-RV & 0.38 & $<0.0001$ \\
CE-RTV-T2/F-RV & 0.22 & $\mathbf{0 . 0 1 2}$ \\
EOR-CE-PTV & 0.04 & 0.677 \\
EOR-CE-RTV & -0.71 & $<0.0001$ \\
EOR-T2/F-RV & -0.07 & 0.401 \\
\hline
\end{tabular}

\footnotetext{
* All volumes were defined from the attending neurosurgeon's mea-
} surements. Boldface type indicates statistical significance.
TABLE 4: Unadjusted analysis of survival predictors*

\begin{tabular}{lc}
\hline \multicolumn{1}{c}{ Variable } & p Value \\
\hline age & $\mathbf{0 . 0 0 7}$ \\
KPS score & $<0.0001$ \\
$\log (\mathrm{PTV})$ & $\mathbf{0 . 0 0 3}$ \\
$\log (\mathrm{RTV})$ & $\mathbf{0 . 0 0 1}$ \\
$\log (\mathrm{T} 2 / \mathrm{F}-\mathrm{RV})$ & $<0.001$ \\
EOR & 0.065 \\
eloquence $\dagger$ & 0.056 \\
\hline
\end{tabular}

\footnotetext{
* All volumes were defined from the attending neurosurgeon's measurements. Boldface type indicates statistical significance.

$\dagger$ Eloquence: noneloquent versus near-eloquent/eloquent as described by Sawaya et al. ${ }^{17}$
}

Univariate predictors of survival are noted in Table 4. The variables age, KPS score, CE-PTV, CE-RTV, and T2/F-RV all achieved statistical significance with $\mathrm{p}<$ 0.05 . EOR $(p=0.07)$ and eloquence $(p=0.06)$ were only marginally significant.

Due to the correlations among variables as noted above, we created separate multivariable models for each volume measurement to identify the best predictor of survival, when controlling for age and KPS score (Table 5). These models indicated that the CE-PTV, CE-RTV, T2/ F-RV and EOR were all statistically significant predictors of survival; however, the volume predictor of survival with the lowest AIC value was determined to be CE-RTV.

CE-RTV and EOR were further analyzed to determine whether useful dichotomized thresholds could be generated (Tables 6 and 7 and Fig. 1). For CE-RTV, statistical significance was achieved with cutoffs in the range of less than $2 \mathrm{~cm}^{3}$ of RTV, with $80(62.5 \%)$ of the 128 patients having had primary resections that achieved this criterion. This range of cutoffs provided hazard ratios of approximately 0.5 , which means that having a residual tumor volume of less than $1-2 \mathrm{~cm}^{3}$ reduces the risk of death by half on average. Alternatively, in regard to EOR, statistical significance was achieved with resections of $98 \%$ or more, with 37 (28.9\%) of the 128 patients in this group.

TABLE 5: Comparison of models associating tumor measurements on survival in patients with $\mathrm{GBM}^{*}$

\begin{tabular}{lccc}
\hline \multicolumn{1}{c}{ Variable } & AIC & HR $(95 \%$ Cl $)$ & p Value \\
\hline PTV & 921.4 & $1.01(0.99-1.01)$ & 0.19 \\
$\log (\mathrm{PTV})$ & 918.2 & $1.25(1.02-1.53)$ & 0.03 \\
RTV & 919.5 & $1.04(1.00-1.08)$ & 0.03 \\
$\log (\mathrm{RTV})$ & 915.8 & $1.42(1.11-1.81)$ & 0.005 \\
EOR $(\%)$ & 918.7 & $0.98(0.95-0.99)$ & 0.03 \\
T2/F-RV & 920.5 & $1.01(0.99-1.01)$ & 0.10 \\
$\log ($ T2/F-RV $)$ & 917.5 & $1.28(1.04-1.57)$ & 0.02 \\
\hline
\end{tabular}

* All models adjusted for patient age and KPS score. All volumes were defined from the attending neurosurgeon's measurements. Boldface type indicates statistical significance. $\mathrm{AIC}=$ Aikake information criterion. 


\section{Residual tumor volume and survival in GBM}

TABLE 6: Comparison of patient survival according to various contrast-enhancing residual tumor volume thresholds*

\begin{tabular}{|c|c|c|c|c|c|c|}
\hline \multirow[b]{2}{*}{$\begin{array}{l}\text { Cut-Point } \\
\qquad\left(\mathrm{cm}^{3}\right)\end{array}$} & \multicolumn{2}{|c|}{ Below Cut-Point } & \multicolumn{2}{|c|}{ Above Cut-Point } & \multirow[b]{2}{*}{$\begin{array}{c}\text { HR }(95 \% \text { Cl) Below } \\
\text { vs Above }\end{array}$} & \multirow[b]{2}{*}{ p Value } \\
\hline & $\begin{array}{l}\text { No. of } \\
\text { Cases }\end{array}$ & $\begin{array}{l}\text { Median Survival } \\
\text { in Mos }(95 \% \mathrm{Cl})\end{array}$ & $\begin{array}{l}\text { No. of } \\
\text { Cases }\end{array}$ & $\begin{array}{l}\text { Median Survival } \\
\text { in Mos (95\% Cl) }\end{array}$ & & \\
\hline 1.00 & 57 & $19(14-29)$ & 71 & $12(10-15)$ & $0.47(0.32-0.70)$ & 0.0002 \\
\hline 1.25 & 65 & $16(13-26)$ & 63 & $13(10-15)$ & $0.52(0.35-0.77)$ & 0.0009 \\
\hline 1.50 & 69 & $16(14-25)$ & 59 & $12(10-14)$ & $0.50(0.34-0.74)$ & 0.0004 \\
\hline 2.00 & 80 & $16(13-20)$ & 48 & $12(10-15)$ & $0.59(0.40-0.86)$ & 0.007 \\
\hline 2.50 & 94 & $15(12-18)$ & 34 & $13(10-16)$ & $0.79(0.52-1.19)$ & 0.26 \\
\hline 3.00 & 97 & $15(13-18)$ & 31 & $13(9-16)$ & $0.77(0.50-1.19)$ & 0.24 \\
\hline 3.50 & 100 & $15(13-18)$ & 28 & $12(8-15)$ & $0.68(0.43-1.07)$ & 0.09 \\
\hline 4.00 & 105 & $15(13-17)$ & 23 & $12(8-15)$ & $0.71(0.44-1.15)$ & 0.16 \\
\hline 4.50 & 109 & $15(13-17)$ & 19 & $12(8-16)$ & $0.65(0.38-1.11)$ & 0.11 \\
\hline 5.00 & 111 & $15(13-17)$ & 17 & $11(8-15)$ & $0.66(0.38-1.16)$ & 0.15 \\
\hline 10.00 & 122 & $14(12-16)$ & 6 & $11(4-31)$ & $0.43(0.18-0.99)$ & 0.05 \\
\hline 15.00 & 124 & $14(12-16)$ & 4 & $11(4-15)$ & $0.36(0.13-1.02)$ & 0.05 \\
\hline 20.00 & 126 & $14(12-16)$ & 2 & $14(14-15)$ & $0.56(0.13-2.36)$ & 0.43 \\
\hline
\end{tabular}

* Boldface type indicates statistical significance.

If the EOR was above $98 \%$, the risk of death at any time was approximately halved when compared with patients with an EOR below $98 \%$.

\section{Predictors of Survival-Interobserver Predictive Strength}

To assess the predictive strength of each assessor's volume measurements on overall survival, multiple multivariable Cox proportional hazards analyses were performed (Tables 8 and 9). For CE-RTV, all assessors' volumes achieved statistical significance, with the attending neurosurgeon's measurement achieving higher significance $(\mathrm{p}=0.005)$ and larger impact, as measured by hazard ratio, when compared with those of the fellow $(\mathrm{p}=0.049)$ and medical student $(\mathrm{p}=0.047)$. In regards to EOR, only the attending neurosurgeon's assessment achieved statistical significance $(\mathrm{p}=0.030)$ with respect to predicting overall survival; those of the fellow and medical student did not achieve statistical significance $(p$ $=0.133$ and $\mathrm{p}=0.122$, respectively).

\section{Discussion}

In this series of 128 consecutive patients undergoing primary resection of GBM followed by standard radiation and chemotherapy, we observed that CE-RTV, T2/F$\mathrm{RV}$, and EOR were predictive of survival, independent of age, disability level, and eloquence. Furthermore, we have demonstrated that CE-RTV was a stronger predic-

TABLE 7: Comparison of patient survival according to various extent of resection thresholds*

\begin{tabular}{|c|c|c|c|c|c|c|}
\hline \multirow[b]{2}{*}{ Cut-Point (\%) } & \multicolumn{2}{|c|}{ Below Cut-Point } & \multicolumn{2}{|c|}{ Above Cut-Point } & \multirow[b]{2}{*}{$\begin{array}{c}\text { HR }(95 \% \text { Cl) Above vs } \\
\text { Below }\end{array}$} & \multirow[b]{2}{*}{$p$ Value } \\
\hline & $\begin{array}{l}\text { No. of } \\
\text { Cases }\end{array}$ & $\begin{array}{c}\text { Median Survival in } \\
\text { Months }(95 \% \mathrm{Cl})\end{array}$ & $\begin{array}{l}\text { No. of } \\
\text { Cases }\end{array}$ & $\begin{array}{c}\text { Median Survival in } \\
\text { Months }(95 \% \mathrm{Cl})\end{array}$ & & \\
\hline 82.00 & 9 & $12(3-19)$ & 119 & $15(13-16)$ & $0.61(0.30-1.26)$ & 0.18 \\
\hline 84.00 & 12 & $14(4-31)$ & 116 & $14(12-16)$ & $1.02(0.53-1.97)$ & 0.95 \\
\hline 86.00 & 19 & $15(8-19)$ & 109 & $14(12-16)$ & $0.85(0.51-1.43)$ & 0.54 \\
\hline 88.00 & 26 & $14(8-18)$ & 102 & $14(12-16)$ & $0.88(0.56-1.40)$ & 0.59 \\
\hline $89 \dagger$ & 32 & $15(10-18)$ & 96 & $14(12-16)$ & $0.81(0.53-1.23)$ & 0.32 \\
\hline 90.00 & 36 & $14(9-16)$ & 92 & $14(12-17)$ & $0.71(0.47-1.07)$ & 0.71 \\
\hline 92.00 & 46 & $15(11-16)$ & 82 & $14(12-19)$ & $0.74(0.50-1.10)$ & 0.13 \\
\hline 94.00 & 56 & $14(11-16)$ & 72 & $14(12-19)$ & $0.71(0.48-1.03)$ & 0.07 \\
\hline 96.00 & 65 & $14(11-16)$ & 63 & $15(12-20)$ & $0.72(0.49-1.04)$ & 0.08 \\
\hline $98 \dagger$ & 91 & $14(12-15)$ & 37 & $16(12-31)$ & $0.65(0.42-0.99)$ & 0.05 \\
\hline 99.00 & 115 & $14(12-16)$ & 13 & $38(11-46)$ & $0.49(0.25-0.96)$ & 0.04 \\
\hline 100.00 & 121 & $14(12-16)$ & 7 & $38(11-\infty)$ & $0.40(0.16-0.99)$ & 0.05 \\
\hline
\end{tabular}

* Boldface type indicates statistical significance.

$\dagger$ Cutoffs identified by Lacroix et al. ${ }^{9}$ 

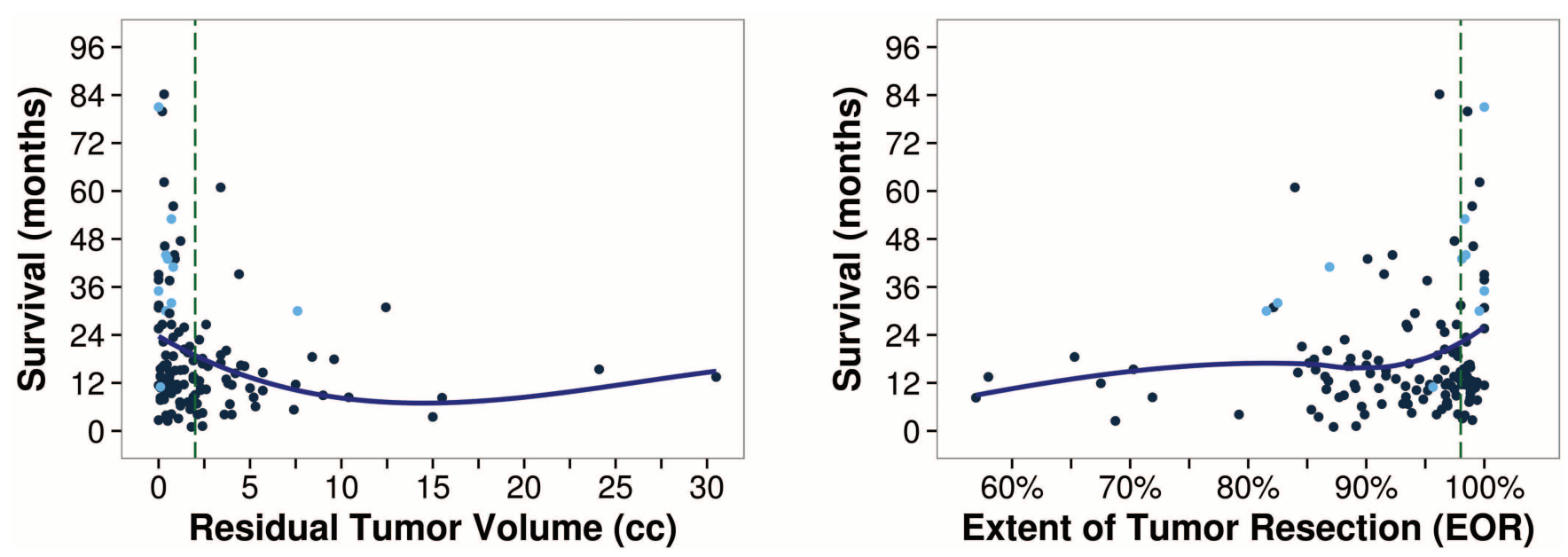

FIG. 1. Association between patient survival and CE-RTV (left) and EOR (right), with locally weighted scatterplot smoothing curves. The dashed lines represent study-identified cutoffs of $2 \mathrm{~cm}^{3}$ of residual tumor volume and $98 \%$ EOR. The 10 patients alive at the time of analysis (censored) are represented as blue points.

tor of survival than EOR or T2/F-RV, with significant survival benefit noted for residual volumes of less than 2 $\mathrm{cm}^{3}$ of contrast-enhancing disease. This is the first study to indicate that CE-RTV is a more important predictor of outcome than EOR, and it validates our hypothesis that residual enhancing tumor burden, prior to the start of medical therapy, is an important prognostic factor for these patients.

We used a quantitative, semi-automated segmentation algorithm to measure CE-RTV. This prototype, advanced version of software that is widely used for neuronavigation is relatively straightforward to deploy, understand, and operate for these purposes, and there was little variability in the measurement of pretreatment tumor volumes between assessors who had a wide degree of experience in neurosurgical oncology (ranging from none to more than a decade). However, the correlation data, along with the predictive strength of each assessor's RTV and EOR measurements, revealed that there was significant variability between measurements of postoperative contrast-enhancing volumes (and hence the ability of each variable to predict a survival benefit), which appears to be dependent upon years of training and experience. The likely cause for this variability (and where experience becomes important) was in the discerning of RTV.

The presence of blood products, among other objects,

TABLE 8: Results from multiple multivariable Cox proportional hazards analyses to assess effect of residual tumor volume on survival in patients with GBM when controlling for age and KPS*

\begin{tabular}{ccc}
\hline Variable & $\mathrm{HR}(95 \% \mathrm{Cl})$ & $\mathrm{p}$ Value \\
\hline MS: $\log (\mathrm{CE}-\mathrm{RTV})$ & $1.27(1.003-1.60)$ & $\mathbf{0 . 0 4 7}$ \\
FEL: $\log (\mathrm{CE}-\mathrm{RTV})$ & $1.23(1.001-1.50)$ & $\mathbf{0 . 0 4 9}$ \\
AN†: $\log (\mathrm{CE}-\mathrm{RTV})$ & $1.42(1.10-1.80)$ & $\mathbf{0 . 0 0 5}$ \\
\hline
\end{tabular}

* Boldface type indicates statistical significance.

$\dagger$ Standard measurement for this study (attending neurosurgeon's measurement). in the region of the resection cavity creates nonspecific T1 (or MPRAGE) hyperintensity that could artificially skew the results. Albert et al. and others have reported enhancement of variable thickness and intensity observed along the margins of resection on MRI within days of surgery, which our study attempted to account for by requiring all postoperative imaging to be obtained no more than 48 hours after surgery.1,23 However, there are other sources of nonspecific enhancement present during the immediate postoperative period, including the choroid plexus, the ependyma, dilated veins at surgical sites, and blood products in the surgical bed. ${ }^{1,8,18,20} \mathrm{We}$ attempted to control for these sources by the comparison of noncontrast to postcontrast postoperative imaging.

While we did not measure variability among assessors of the same experience level, other studies have investigated interobserver agreement with results similar to ours. ${ }^{11}$ It is apparent that our measurement methodology was a limitation of the study, as the data indicate that the assessor's experience remains important in the evaluation of postoperative scans. These limitations may be overcome utilizing work by Kanaly and colleagues, whose nearly fully automated software algorithm was designed to minimize interobserver variability while accounting for factors such as resection cavity collapse and the presence of blood products after surgery. ${ }^{9}$ However, it should

TABLE 9: Results from multiple multivariable Cox proportional hazards analyses to assess effect of EOR on survival in patients with GBM when controlling for age and KPS*

\begin{tabular}{ccc}
\hline Variable & HR $(95 \% \mathrm{Cl})$ & p Value \\
\hline MS: EOR & $0.28(0.06-1.51)$ & 0.134 \\
FEL: EOR & $0.33(0.10-1.37)$ & 0.123 \\
AN†: EOR & $0.08(0.01-0.84)$ & 0.030 \\
\hline
\end{tabular}

\footnotetext{
* Boldface type indicates statistical significance.

$\dagger$ Standard measurement for this study (attending neurosurgeon's measurement).
} 
be noted that ours is the first study to report assessors' experience as a factor to be considered when evaluating the utility of a particular volumetric approach. Future studies assessing tumor volume should be as automated as possible to standardize measurements across assessors of both varying degrees of experience and from different institutions.

This study also looked at the predictive value of residual volume of FLAIR abnormality. Although tumor infiltration into normal brain has been observed in nonenhancing areas, there are questions regarding its usefulness and timing in delineating tumor burden and predicting survival. ${ }^{10}$ In our patient population, T2/F-RV is a significant predictor of survival in both univariate and multivariable analyses. However, preoperative mass effect on surrounding tissue, along with intraoperative manipulation of tissue within the surgical corridor and tumor bed can create an abnormal, edema-related T2/ FLAIR signal that persists for days to weeks after surgery. ${ }^{21}$ Consequently, an unknown portion of the measured abnormal T2/F-RV may represent edema that will eventually resolve, rather than tumor infiltration. Thus this may represent a methodological shortcoming of our study, since the FLAIR images were obtained less than 48 hours after surgery.

Previous studies have suggested an association between EOR and both time to progression and survival. One of the first studies to examine this association was by Lacroix et al., who performed a retrospective analysis of 416 GBM cases in which EOR was determined by comparing findings on contrast-enhancing preoperative and postoperative MRI using computerized volumetric analysis. ${ }^{12}$ The authors reported a significant survival advantage when EOR was $98 \%$ or greater. Their methodology was innovative and superior to studies previously performed due to the standardized, computer-based quantification of tumor volume; however, their analysis differed from ours specifically with regard to the measurement of RTV. RTV alone was not analyzed to determine if it was a predictor of outcome. Albert et al. looked prospectively at the postoperative MRI studies in 60 cases in which patients underwent resection of high-grade gliomas. ${ }^{1}$ They found that those patients with residual contrast-enhancing tumor had approximately 6.6 times the risk of death as those patients without residual tumor. However, residual tumor was defined qualitatively as definite enhancement, uncertain findings, and without enhancement. Thus, the quantitative effect of RTV remained unclear. The largest study was performed by Sanai et al., who assessed 500 consecutive cases of newly diagnosed GBM and found that an EOR of $78 \%$ or greater positively impacted patient survival, with this trend continuing toward higher EOR percentages. ${ }^{16}$ In addition, within a multivariable Cox proportional hazards analysis, they found RTV to be highly predictive of survival, along with age, KPS, and EOR. However, the significance of impact of RTV on survival within their model was not discussed.

While our results support previously published data regarding the significance of EOR and its association with survival, they more importantly underscore the significance of RTV in predicting survival. The EOR and
RTV thresholds we found for predicting overall survival differ from those recently identified by Chaichana et al. ${ }^{6}$ The authors found that an EOR threshold of $70 \%$ and a RTV threshold of $5 \mathrm{~cm}^{3}$ were statistically associated with survival, which is a lower EOR and higher RTV threshold than ours. The causes for this divergence in results are unclear, but it may be related to the differences in the patient populations studied. While the median overall survival in their study was relatively similar to that in ours (13.4 vs 13.8 months, respectively) and the median PTV was also $\left(32.2\right.$ vs $\left.29.0 \mathrm{~cm}^{3}\right)$, their patients' median RTV was nearly twice that of ours (2.1 vs $\left.1.2 \mathrm{~cm}^{3}\right)$. Since the overall survival and PTV were relatively similar, it stands to reason that Chaichana et al. would find a higher RTV threshold in their study since their median RTV was higher. Accordingly, the lower RTVs observed in our patients provided us with a greater sensitivity to detect small differences in outcome as a function of RTV.

Although this volume of work has produced considerable evidence that greater EOR increases time to survival, the results are tempered by the fact that the analysis of the impact of EOR has been performed in retrospect only and not by prospective design. Being retrospective in nature, our study suffers from the same shortcomings as the previous literature, specifically with regard to selection bias, lack of randomization, and difficulty in controlling for confounders and establishing cause and effect. However, there are understandable ethical and practical concerns that have contributed to the lack of rigorous Level 1 evidence regarding the relationship between EOR and outcome. For example, it would be of dubious ethical probity to randomize patients with GBM to an intended subtotal resection, and it is unlikely that patients would agree to such a study even if approved by an institutional review board. While prospective data collection is feasible and would improve study design, the other limitations are considerably more difficult to overcome and thus have constrained the ability of the neurosurgical community to causally link surgical outcome, defined by completeness of resection, with progression and overall survival. Nevertheless, this ethical dilemma is mitigated by the substantial data that support the practice of extensive removal of enhancing tumor tissue, limited primarily by proximity to eloquent brain, and this goal remains the widely accepted standard of care for patients with newly diagnosed GBM.

Since EOR is defined, in large part, by RTV, the correlation of the two measures EOR and RTV is expected since they quantify two related aspects of tumor volume and the impact of surgery. Notably, our data show that PTV and EOR are not correlated with one another, suggesting that a surgeon is just as likely to achieve a high EOR or a low EOR with any given PTV. Since the actual variable that has an association with the outcome and is driving the EOR calculation is RTV, one may question the necessity of including PTV in EOR as a predictor of survival. While our results do not diminish the value of EOR demonstrated in previously published studies, they suggest that the benefit seen with more extensive EORs largely arises from the consequent small RTVs that were obtained, but not fully analyzed. 
In addition to statistical evidence, there are other practical arguments to be made that support minimization of RTV to be the more useful and pragmatic stated goal for surgery than increasing EOR. For example, a patient with a $25-\mathrm{cm}^{3}$ PTV who undergoes resection and has an EOR of $90 \%$ would have a significantly larger RTV than a patient with a $5-\mathrm{cm}^{3}$ PTV with EOR of $90 \%$ (RTV 2.5 vs. $0.5 \mathrm{~cm}^{3}$, respectively), and it is not clear that an emphasis on EOR will convey this biological reality to clinicians or patients. Based on the results of this study, neither patient would be predicted to have attained a survival benefit solely utilizing EOR as the predictor, while the patient with a RTV of $0.5 \mathrm{~cm}^{3}$ would be predicted to have attained a survival benefit using RTV. From a practical standpoint, it is difficult to assess EOR intraoperatively, even with the use of intraoperative imaging techniques, and the difference between 5\% increments in EOR may not have practical relevance in the preoperative or intraoperative setting. RTV may be a more practical measure, especially when intraoperative imaging is employed. One would expect that the CE-RTV goal for GBM should be $0 \mathrm{~cm}^{3}$, but we found that a CE-RTV of $2 \mathrm{~cm}^{3}$ or less still confers survival benefit to the patient. While the exact reasons for this effect are not clear, we speculate (along with others ${ }^{16}$ ) that the residual tumor's response to subsequent radiation and chemotherapy may be nonlinear, with smaller, scattered RTVs having a more favorable response. Given the results and arguments outlined here, we believe that the pathobiology of GBM is primarily dependent on RTV, not EOR, and that RTV is the more clinically relevant measure.

\section{Conclusions}

When measured volumetrically, CE-RTV, CE-PTV, T2/F-RV, and EOR were all found to be significant predictors of survival after GBM resection when controlling for age and KPS score. In our study, contrast-enhancing residual tumor volume (CE-RTV) was the most significant predictor of survival when compared with EOR, T2/ FLAIR residual volume (T2/F-RV), and contrast-enhancing preoperative tumor volume (CE-PTV), which suggests that residual tumor volume may be a more accurate and meaningful reflection of the pathobiology of these tumors. Future studies are needed to further clarify the effect of CE-RTV on patient survival after GBM resection.

\section{Acknowledgments}

The authors would like to thank Stephan Mittermeyer, Rowena Thomson, and Elizabeth Vasconcellos of BrainLAB AG for providing access to and support for a prototype version of their iPLAN, which has advanced tools that were used for volumetric analysis.

\section{Disclosure}

No financial support was provided for this study. M.A.V. is supported by the Wolf Family Foundation and the Robert and Kathryn Lamborn Chair in Neuro-Oncology.

Author contributions to the study and manuscript preparation include the following. Conception and design: Vogelbaum, Grabowski. Acquisition of data: Vogelbaum, Grabowski, Recinos, Schroeder. Analysis and interpretation of data: Vogelbaum,
Grabowski, Nowacki. Drafting the article: Grabowski. Critically revising the article: all authors. Reviewed submitted version of manuscript: all authors. Approved the final version of the manuscript on behalf of all authors: Vogelbaum. Statistical analysis: Nowacki. Study supervision: Vogelbaum.

\section{References}

1. Albert FK, Forsting M, Sartor K, Adams HP, Kunze S: Early postoperative magnetic resonance imaging after resection of malignant glioma: objective evaluation of residual tumor and its influence on regrowth and prognosis. Neurosurgery 34:4561,1994

2. Bloch O, Han SJ, Cha S, Sun MZ, Aghi MK, McDermott MW, et al: Impact of extent of resection for recurrent glioblastoma on overall survival. Clinical article. J Neurosurg 117:10321038,2012

3. Buckner JC: Factors influencing survival in high-grade gliomas. Semin Oncol 30 (6 Suppl 19):10-14, 2003

4. Central Brain Tumor Registry of the United States: CBTRUS Statistical Report: Primary Brain and Central Nervous System Tumors Diagnosed in the United States in 20042006. Hinsdale, IL: Central Brain Tumor Registry of the United States, 2010 (http://www.cbtrus.org/2010-NPCR-SEER/ CBTRUS-WEBREPORT-Final-3-2-10.pdf) [Accessed July 23, 2014]

5. Chaichana KL, Chaichana KK, Olivi A, Weingart JD, Bennett $\mathrm{R}$, Brem H, et al: Surgical outcomes for older patients with glioblastoma multiforme: preoperative factors associated with decreased survival. Clinical article. J Neurosurg 114:587-594, 2011

6. Chaichana KL, Jusue-Torres I, Navarro-Ramirez R, Raza SM, Pascual-Gallego M, Ibrahim A, et al: Establishing percent resection and residual volume thresholds affecting survival and recurrence for patients with newly diagnosed intracranial glioblastoma. Neuro Oncol 16:113-122, 2014

7. Dea N, Fournier-Gosselin MP, Mathieu D, Goffaux P, Fortin $\mathrm{D}$ : Does extent of resection impact survival in patients bearing glioblastoma? Can J Neurol Sci 39:632-637, 2012

8. Elster AD, DiPersio DA: Cranial postoperative site: assessment with contrast-enhanced MR imaging. Radiology 174: 93-98, 1990

9. Kanaly CW, Ding D, Mehta AI, Waller AF, Crocker I, Desjardins A, et al: A novel method for volumetric MRI response assessment of enhancing brain tumors. PLoS ONE 6:e16031, 2011

10. Kelly PJ, Daumas-Duport C, Kispert DB, Kall BA, Scheithauer BW, Illig JJ: Imaging-based stereotaxic serial biopsies in untreated intracranial glial neoplasms. J Neurosurg 66:865874, 1987

11. Kubben PL, Postma AA, Kessels AG, van Overbeeke JJ, van Santbrink H: Intraobserver and interobserver agreement in volumetric assessment of glioblastoma multiforme resection. Neurosurgery 67:1329-1334, 2010

12. Lacroix M, Abi-Said D, Fourney DR, Gokaslan ZL, Shi W, DeMonte F, et al: A multivariate analysis of 416 patients with glioblastoma multiforme: prognosis, extent of resection, and survival. J Neurosurg 95:190-198, 2001

13. McGirt MJ, Chaichana KL, Gathinji M, Attenello FJ, Than K, Olivi A, et al: Independent association of extent of resection with survival in patients with malignant brain astrocytoma. Clinical article. J Neurosurg 110:156-162, 2009

14. Orringer D, Lau D, Khatri S, Zamora-Berridi GJ, Zhang K, $\mathrm{Wu} \mathrm{C}$, et al: Extent of resection in patients with glioblastoma: limiting factors, perception of resectability, and effect on survival. Clinical article. J Neurosurg 117:851-859, 2012

15. Pang BC, Wan WH, Lee CK, Khu KJ, Ng WH: The role of surgery in high-grade glioma-is surgical resection justified? 


\section{Residual tumor volume and survival in GBM}

A review of the current knowledge. Ann Acad Med Singapore 36:358-363, 2007

16. Sanai N, Polley MY, McDermott MW, Parsa AT, Berger MS: An extent of resection threshold for newly diagnosed glioblastomas. Clinical article. J Neurosurg 115:3-8, 2011

17. Sawaya R, Hammoud M, Schoppa D, Hess KR, Wu SZ, Shi WM, et al: Neurosurgical outcomes in a modern series of 400 craniotomies for treatment of parenchymal tumors. Neurosurgery 42:1044-1056, 1998

18. Sinclair AG, Scoffings DJ: Imaging of the post-operative cranium. Radiographics 30:461-482, 2010

19. Stupp R, Hegi ME, Mason WP, van den Bent MJ, Taphoorn MJB, Janzer RC, et al: Effects of radiotherapy with concomitant and adjuvant temozolomide versus radiotherapy alone on survival in glioblastoma in a randomised phase III study: 5-year analysis of the EORTC-NCIC trial. Lancet Oncol 10:459-466, 2009

20. Sze G: New applications of MR contrast agents in neuroradiology. Neuroradiology 32:421-438, 1990

21. Vogelbaum MA, Jost S, Aghi MK, Heimberger AB, Sampson
JH, Wen PY, et al: Application of novel response/progression measures for surgically delivered therapies for gliomas: Response Assessment in Neuro-Oncology (RANO) Working Group. Neurosurgery 70:234-244, 2012

22. Wen PY, Kesari S: Malignant gliomas in adults. N Engl J Med 359:492-507, 2008

23. Wen PY, Macdonald DR, Reardon DA, Cloughesy TF, Sorensen AG, Galanis E, et al: Updated response assessment criteria for high-grade gliomas: Response Assessment in NeuroOncology Working Group. J Clin Oncol 28:1963-1972, 2010

Manuscript submitted November 5, 2013.

Accepted July 22, 2014.

Please include this information when citing this paper: published online September 5, 2014; DOI: 10.3171/2014.7.JNS132449.

Address correspondence to: Michael A. Vogelbaum, M.D., Ph.D., Department of Neurosurgery, Cleveland Clinic, 9500 Euclid Ave., Cleveland, $\mathrm{OH}$ 44195. email: vogelbm@ ccf.org. 